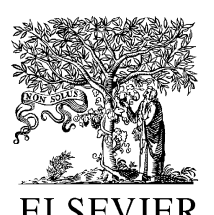

\title{
The necessity for multiphoton processes in the 193-nm photochemistry of sulphuric acid aerosols
}

\author{
S.J. Wrenn ${ }^{\text {a }}$, L.J. Butler ${ }^{\text {a }}$, G.A. Rowland ${ }^{\text {b }}$, C.J.H. Knox ${ }^{\text {b }}$, L.F. Phillips ${ }^{\text {b,* }}$ \\ a The James Franck Institute and the Department of Chemistry, The University of Chicago, Chicago, IL 60637, USA \\ ${ }^{\mathrm{b}}$ Chemistry Department, University of Canterbury, Christchurch, New Zealand
}

Received 21 June 1999; received in revised form 26 July 1999; accepted 5 August 1999

\begin{abstract}
The observation, that absorption of light by concentrated sulphuric acid at wavelengths near $193 \mathrm{~nm}$ apparently tends to zero as the acid is progressively purified, is supported by ab initio calculations of the energies of the three lowest excited states of monomeric $\mathrm{H}_{2} \mathrm{SO}_{4}$. The electronic-structure calculations indicate that the lowest excited state is not accessible by single-photon absorption for light of wavelengths $>150 \mathrm{~nm}$. This limit includes a generous allowance for error; the calculated energy of the transition corresponds to a wavelength $<120 \mathrm{~nm}$. Previous observations of photoluminescence and photo-oxidation of $\mathrm{CO}$ in sulphuric acid aerosols are attributed to multiphoton processes, caused by internal focusing of the incident light by the aerosol droplets. This finding does not rule out the occurrence of significant photochemistry of sulphuric acid aerosols in the stratospheres of the Earth and Venus, because of the likely presence of transition-metal ions as impurities in the atmospheric aerosols. (1999 Elsevier Science S.A. All rights reserved.
\end{abstract}

Keywords: Sulphuric acid absorption spectrum; Sulphuric acid photochemistry; Sulphuric acid excited states; Sulphuric acid aerosols; Multiphoton effects in aerosols

\section{Introduction}

The photochemistry of tropospheric aerosols containing dilute sulphurous and sulphuric acids plus traces of transition-metal ions has been shown to be unexpectedly rich and diverse [1]. Aerosols consisting largely of concentrated sulphuric acid are present in the upper atmospheres of Earth and Venus, and it seems reasonable to assume that the chemical consequences of ultraviolet irradiation of these aerosols should be included in atmospheric models. Previously, photoluminescence [2] and photo-oxidation of $\mathrm{CO}$ to $\mathrm{CO}_{2}[3,4]$ have been observed in sulphuric acid aerosols irradiated with unfocused $193 \mathrm{~nm}$ radiation from an $\mathrm{ArF}$ laser, and these observations were attributed to secondary processes following single-photon absorption at $193 \mathrm{~nm}$. However, difficulty was encountered in identifying the primary process, and our subsequent attempts to measure the amount of light absorption by concentrated sulphuric acid near $193 \mathrm{~nm}$ have been frustrated by the observation that this absorption, which is initially very weak for analytical reagent grade acid, tends towards zero as the acid is progressively freed of dissolved gases by pumping. We pre-

\footnotetext{
${ }^{*}$ Corresponding author. Tel.: +39-75-5851; fax: +39-75-585-2067

E-mail address: philips@chem.canterbury.ac.nz (L.F. Phillips)
}

sume that the significant impurity which is being removed is sulfur dioxide, in which case this absorption should not have been important for the aerosols in Refs [1-3], because they were generated by mixing gaseous sulfur trioxide with excess water vapor in the absence of $\mathrm{SO}_{2}$. The theoretical work described here was prompted by the two uncertainties regarding the extent of light absorption by pure $\mathrm{H}_{2} \mathrm{SO}_{4}$ at $193 \mathrm{~nm}$ and the nature of the primary photodissociation process following light absorption.

This paper presents new ab initio electronic structure calculations of the excited electronic states of monomeric $\mathrm{H}_{2} \mathrm{SO}_{4}$. It is obvious that electronic-structure calculations for clusters and agglomerations of $\mathrm{H}_{2} \mathrm{SO}_{4}$ molecules in the liquid phase are needed for a proper understanding of experiments on the photochemistry of $\mathrm{H}_{2} \mathrm{SO}_{4}$ aerosols. Nevertheless, because the results of our calculations for the monomer are unequivocal as far as the question of singlet-singlet absorption by sulphuric acid at $193 \mathrm{~nm}$ is concerned, and because there are no previously published results for the excited states of the monomer, we believe that the present calculations represent a useful starting point and are worth publishing at this stage.

Both, semi-empirical and ab initio methods have been used previously to investigate the $\mathrm{H}_{2} \mathrm{SO}_{4}$ molecule in its ground electronic state [5-11]. Although geometry 
optimization was the primary aim of most of the previous work, results relating to torsional modes [8] and electronic properties [11] were also reported. Early semi-empirical $\mathrm{CNDO} / 2$ calculations predicted overall $\mathrm{C}_{2 \nu}$ symmetry for the molecule [5] and were consistent with existing assumptions about its geometry. However, Kuczkowski et al. [12] showed experimentally that the $\mathrm{H}_{2} \mathrm{SO}_{4}$ molecule belongs to the $\mathrm{C}_{2}$ point group, and that not even the sulfur-oxygen framework of the molecule can be characterized as $C_{2} v$. More recent theoretical work has given results in accord with these experiments $[7,9,10,11]$ and there is now a general agreement as to the molecule's structure in the ground state. So far, no calculations have been reported for excited electronic states of the $\mathrm{H}_{2} \mathrm{SO}_{4}$ molecule, and experimental data for the excited states is non-existent. There have been several attempts to measure the UV absorption of gas-phase monomeric sulphuric acid, motivated by the fact that some atmospheric models have involved photochemistry of $\mathrm{H}_{2} \mathrm{SO}_{4}$ at wavelengths $>150 \mathrm{~nm}$, but the only definite result we are aware of is that of Burkholder et al. [13], who found that the molecular absorption cross section of $\mathrm{H}_{2} \mathrm{SO}_{4}$ in the gas phase is $<10^{-21} \mathrm{~cm}^{2}$ molecule ${ }^{-1}$ at wavelengths $>195 \mathrm{~nm}$. Here, we report the first excited-state calculations for the $\mathrm{H}_{2} \mathrm{SO}_{4}$ molecule, where our findings are in accord with the work of Burkholder et al, and some implications of our results will be discussed.

\section{Method}

We used the Gaussian 94 program [14] to run ab initio calculations with a $6-31 \mathrm{G}^{*}$ basis set of atomic orbitals. Configuration interaction was included for single excitations (CIS), and the calculations were restricted to singlet states. The $\mathrm{C}_{2}$ geometry adopted for these calculations was the experimental one, determined by Kuczkowski et al. [12] We used the OpenMol program [15] to visualize the orbitals generated by the calculations.

\section{Results}

The MP2 total energy computed for the ground-state $\mathrm{H}_{2} \mathrm{SO}_{4}$ molecule with the specified $\mathrm{C}_{2}$ geometry was $-698.89402 \mathrm{au}$. We qualitatively characterize the lowest singlet excited state as being accessed primarily by a nonbonding $\rightarrow \sigma^{*}$ transition, where the antibonding $\sigma^{*}$ character is localized on the $\mathrm{O}-\mathrm{H}$ orbitals. Fig. 1 shows a visualization of the dominant orbitals involved in the transition. For this first excited state, our calculations give a vertical excitation energy of $11.1775 \mathrm{eV}$ (from the $\mathrm{C}_{2}$ ground state at the experimental geometry of Kuczkowski et al. [12]) and an oscillator strength $f=0.0046$. Fig. 2 shows the two strongest one-electron excitations in the configuration interaction expansion contributing to the second excited state. The vertical transition energy is $11.2592 \mathrm{eV}$, with an
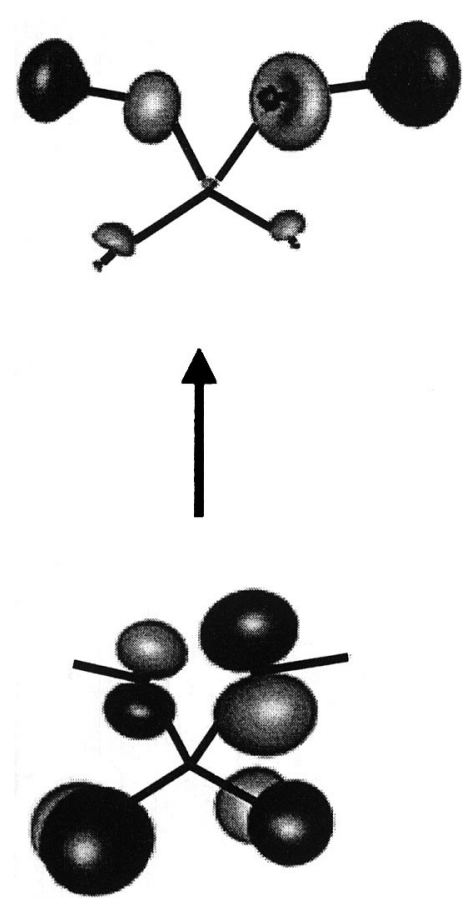

Fig. 1. Visualization of the strongest one-electron excitation contributing to the first excited state of $\mathrm{H}_{2} \mathrm{SO}_{4}$ given by CIS calculations. We qualitatively characterize the transition as nonbonding $\rightarrow \sigma^{*}$. Note that the excited state antibonding character is localized on the $\mathrm{O}-\mathrm{H}$ portions of the molecule.

oscillator strength $f=0.0093$. We also characterize this transition as nonbonding $\rightarrow \sigma^{*}$, this time with both, $\mathrm{O}-\mathrm{H}$ and $\mathrm{S}-\mathrm{O}$ having an antibonding character. Our calculations give an oscillator strength of zero for the transition to the third excited state, so it is regarded as inaccessible. Transitions to higher excited states require a vertical excitation energy of $11.7782 \mathrm{eV}$ or more. A summary of results for the $\mathrm{C}_{2}$ conformer is given in Table 1.

Two important limitations apply to our computed results. First, the CIS method cannot predict any excited state whose dominant electronic character corresponds to a two-electron excitation from the ground-state reference configuration. However, such states would not have a large oscillator strength for direct excitation from the ground state. The second limitation relates to the accuracy of the calculated vertical excitation energies. The CIS method gives excitation energies that typically are in error by (1-2) eV, and occasionally by as much as $3 \mathrm{eV}$. Our reported values should, therefore, be regarded as having a maximum uncertainty of $\pm 3 \mathrm{eV}$.

Before Kuczkowski et al. [12] convincingly demonstrated the $\mathrm{C}_{2}$ nature of sulphuric acid's structure, it was thought that the overall symmetry of the molecule was $C_{2 v}$ [16-19]. If there were a significant equilibrium population of molecules with $\mathrm{C}_{2 v}$ symmetry, then vertical transitions from the hypothetical $\mathrm{C}_{2} v$ conformer of $\mathrm{H}_{2} \mathrm{SO}_{4}$ could give rise to significant absorption. However, our calculations indicate that the $\mathrm{C}_{2 v}$ conformer of Holland and Castleman [5] is about $170 \mathrm{~kJ} / \mathrm{mol}$ higher in energy than Kuczkowski's $\mathrm{C}_{2}$ conformer. This is well beyond the reach of thermal excitation, 


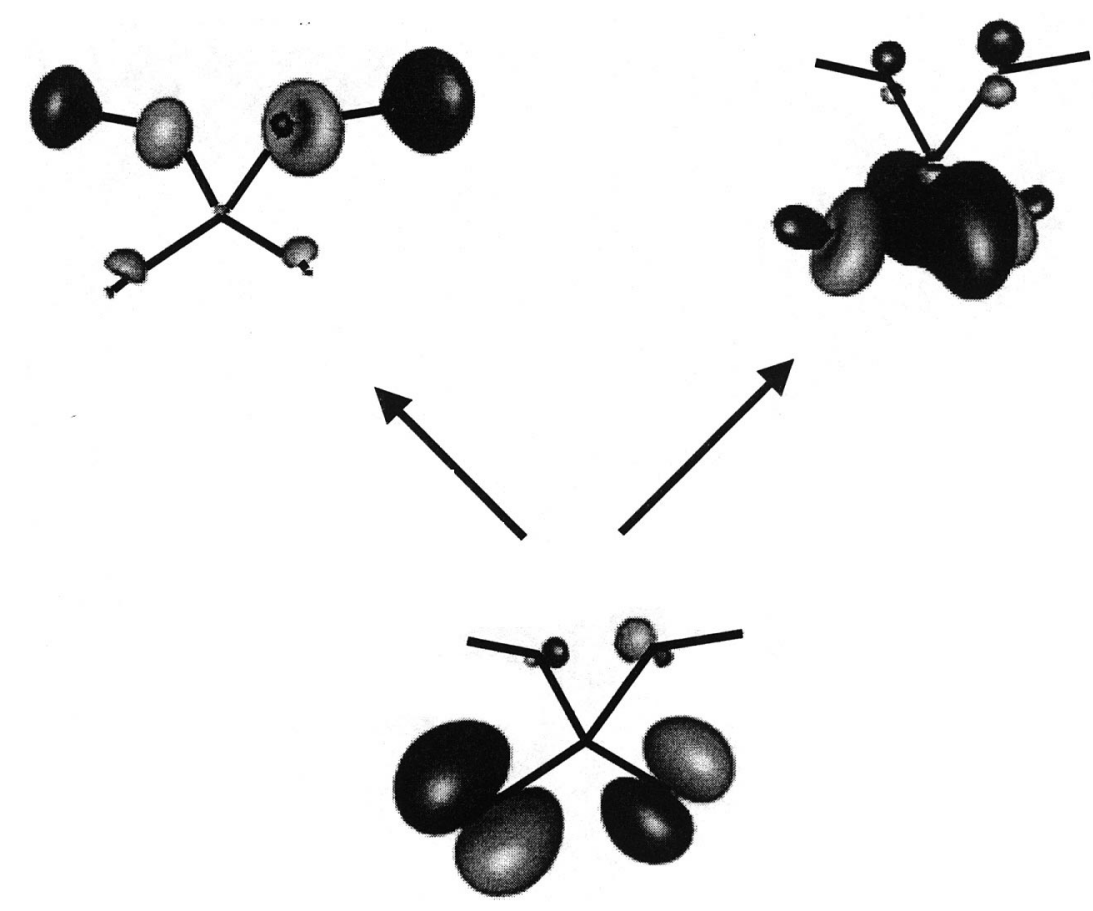

Fig. 2. Visualization of the two strongest one-electron excitation contributing to the second excited state of $\mathrm{H}_{2} \mathrm{SO}_{4}$ given by CIS calculations. We qualitatively characterize the transition as nonbonding $\rightarrow \sigma^{*}$. Note that the excited state antibonding character is localized on the O-H and S-O portions of the molecule.

Table 1

Excited State ab initio calculation results for $\mathrm{C}_{2}$ conformer of $\mathrm{H}_{2} \mathrm{SO}_{4}{ }^{\mathrm{a}}$

\begin{tabular}{llll}
\hline Excited state & Transition energy $(\mathrm{eV})$ & Oscillator strength $(\mathrm{IT})$ & Transition character \\
\hline 1 & 11.1775 & 0.0046 & nonbonding $\rightarrow \sigma^{*}(\mathrm{O}-\mathrm{H})$ \\
2 & 11.2592 & 0.0093 & nonbonding $\rightarrow \sigma^{*}(\mathrm{O}-\mathrm{H}, \mathrm{S}-\mathrm{O})$ \\
3 & 11.5916 & 0.0000 & nonbonding $\rightarrow \sigma^{*}(\mathrm{O}-\mathrm{H} . \mathrm{S}-\mathrm{O})$ \\
4 & 11.7782 & 0.0170 & nonbonding $\rightarrow \sigma^{*}(\mathrm{O}-\mathrm{H})$ \\
\hline
\end{tabular}

${ }^{\text {a }}$ State geometry used was that reported by Kuczowski et al [12].

so absorption by the $\mathrm{C}_{2} v$ species is not likely to be important, either in the laboratory or in planetary atmospheres. Here, we present results only for the experimental $\mathrm{C}_{2}$ structure.

\section{Discussion}

The results of our calculations are consistent with the experimental finding of Burkholder et al., [13]. The vertical excitation energy of $11.1775 \mathrm{eV}$ for the first excited state of the experimentally observed $\mathrm{C}_{2}$ conformer corresponds to light of wavelength $110.92 \mathrm{~nm}$, so this lowest lying singlet excited state is not energetically accessible at the 193-nm laser wavelength. Even if we assume that the calculated transition energy is too large by $3 \mathrm{eV}$, we still find that light of wavelength $<150 \mathrm{~nm}$ is required to access the first excited singlet state. Thus, it is clear that single-photon absorption via a spin-allowed transition cannot initiate photochemistry in pure sulphuric acid vapor irradiated at $193 \mathrm{~nm}$. The occurrence of significant singlet-triplet absorption at $193 \mathrm{~nm}$ can probably be ruled out on the theoretical grounds that, for such absorption to be observable as it is in the case of $\mathrm{SO}_{2}$, the triplet state would need to be close enough in energy to the lowest singlet state for the two states to mix, but the calculated energy of the singlet state is much too high for such a mixed state to be accessible at $193 \mathrm{~nm}$. This argument is weakened when the uncertainty of $\pm 3 \mathrm{eV}$ is applied to the calculated energy, but is not invalidated. We stress that this result may not be trivially extended to the behavior of $\mathrm{H}_{2} \mathrm{SO}_{4}$ in aerosol droplets. Condensed-phase calculations are needed to put the matter entirely beyond doubt. Nevertheless, it seems highly unlikely that liquid-phase interactions could generate the sort of red-shift needed to produce absorption at $193 \mathrm{~nm}$, and this is consistent with our experimental observation that pure, liquid $\mathrm{H}_{2} \mathrm{SO}_{4}$ does not absorb significantly at $\lambda \approx 195 \mathrm{~nm}$ (molar base-1O absorption coefficient $\epsilon<0.5$ at the bottom end of the wavelength range of a standard laboratory spectrophotometer; Burkholder et al's gas-phase upper limit for the cross section corresponds to $\epsilon<0.3)$.

Next, we consider the primary photochemical process that follows excitation. As already noted, the first excited state 
of $\mathrm{H}_{2} \mathrm{SO}_{4}$ involves significant antibonding character on both $\mathrm{O}-\mathrm{H}$ bonds of the molecule. Within the Franck-Condon region, we would expect the excited potential energy surface to be repulsive along the $\mathrm{O}-\mathrm{H}$ bond coordinates, and the forces exerted along these coordinates should lead to an initial lengthening of these bonds. Assuming that the resulting trajectory evolves diabatically along one $\mathrm{O}-\mathrm{H}$ coordinate (i.e. the initial antibonding electronic character is maintained as the bond lengthens), we would therefore expect dissociation to the products $\mathrm{H}+\mathrm{HSO}_{4}$. However, in a molecule as small as $\mathrm{H}_{2} \mathrm{SO}_{4}$, diabaticity cannot be assured, and dissociation via other product channels might also occur.

For both of the allowed transitions considered here, the excitation process corresponds to transfer of one electron from an orbital whose symmetry species is $b$ to one of symmetry species $a$,so the overall transition is $\mathrm{B} \leftarrow A$.In point-group $\mathrm{C}_{2}$, these transitions are allowed for both one-photon and two-photon absorptions. However, two-photon absorption at $193 \mathrm{~nm}$ corresponds to an excitation energy of $12.85 \mathrm{eV}$. At this level, other excited states will be involved, so the nature of the primary process in this case remains unknown even for neutral fragmentation, and photoionization is also a likely outcome, the measured ionization potential being $12.4 \pm 0.5 \mathrm{eV}[20]$.

Two-photon absorption can occur even with unfocused laser radiation if there are 'hot spots' in the beam, but the presence of aerosol droplets in the system of interest makes it unnecessary for us to invoke this mechanism. Chang et al., [21] have described the processes leading to laser-induced breakdown in large (radius ca. $30 \mu \mathrm{m}$, which is large by aerosol standards), transparent water droplets irradiated by visible light. Much of their discussion can be carried over virtually unchanged to our present system, where the droplet radius is of the order of $1 \mu \mathrm{m}$. At our shorter laser wavelength, the mean droplet radius for effective internal focusing is smaller in proportion, and the condition for effective focusing is less stringent because the energy deposited in the droplets does not have to be sufficient to generate a plasma by dielectric breakdown. All that is required is that the local intensity be sufficient to produce multiphoton absorption. An important practical conclusion here is that workers on aerosol photochemistry should refrain from using excimer lasers unless they are interested in the consequences of multiphoton processes.

A point of interest in relation to the original studies of photochemistry of sulphuric acid aerosols was their likely relevance to the upper atmospheres of the Earth and Venus. The conclusion that this work involved multiphoton absorption implies that experiments on pure sulphuric acid aerosols irradiated at $193 \mathrm{~nm}$ have no relevance whatever to planetary atmospheres. However, preliminary work in our laboratory has shown that traces of iron salts in the acid give rise to significant absorption at both 193 and $248 \mathrm{~nm}$, and that photo-oxidation of $\mathrm{Fe}^{\mathrm{II}}$ to $\mathrm{F}^{\mathrm{III}}$ occurs readily in such systems. Transition metals arrive in the Earth's stratosphere in significant amounts as a consequence of meteor ablation
[22], and there is evidence for the presence of iron salts at lower levels in the clouds of Venus [23], in which case the photo-oxidation of $\mathrm{Fe}^{\mathrm{II}}$ to $\mathrm{Fe}^{\mathrm{III}}$ might help to account for the observed very low abundance of $\mathrm{O}_{2}$ above the clouds. Further work is in progress.

\section{Acknowledgements}

This work was supported in the USA by the National Science Foundation under grant No. CHE-96 19376 and by the Joint Institute for Laboratory Astrophysics through their appointment of L.J. Butler as a JILA Visiting Fellow, and in New Zealand it was supported by the Marsden Fund. L.J. Butler would like to thank V. Vaida for helpful discussions and C. Lineberger for hosting her sabbatical leave.

\section{References}

[1] P. Warneck (Ed), Transport and Chemical Transformations of Pollutants in the Troposphere, vol. 2, Heterogeneous and Liquid Phase Processes, Springer-Verlag, Berlin, 1996.

[2] Y.G. Jin, H.C. Zhou, M. Suto, L.C. Lee, J. Photochem. Photobiol. A. Chem. 52 (1990) 225.

[3] C.T. Mills, L.F. Phillips, J. Photochem. Photobiol. A. Chem. 74 (1993) 7.

[4] C.T. Mills, G.A. Rowland, J. Westergren, L.F. Phillips, I. Photochem. Photobiol. A. Chem. 93 (1996) 83.

[5] P.M. Holland, A.W. Castleman Jr., Chem. Phys. Lett. 56 (1978) 511.

[6] N.C. Baird, K.F. Taylor, J. Comput. Chem. 2 (1981) 225.

[7] L.L. Lohr Jr., J. Mol. Struct. 87 (1982) 221.

[8] P. Kaliannan, S. Vishveshwara, V.S.R. Rao, J. Mol. Struct. 92 (1983) 7.

[9] C.H. Patterson, R.P. Messmer, J. Am. Chem. Soc. 112 (1990) 4138.

[10] I. Alkorta, Theor. Chim. Acta 89 (1994) 1.

[11] M. Hofmann, P. von Ragué Schleyer, J. Am. Chem. Soc. 116 (1994) 4947.

[12] R.L. Kuczkowski, R.D. Suenram, F.J. Lovas, J. Am. Chem. Soc. 103 (1981) 2561.

[13] J. Burkholder, M. Mills, S. McKeen, to be published.

[14] GAUSSIAN 94 (Revision E.2), M.J. Frisch, G.W. Trucks, H.B. Schlegel, P.M.W. Gill, B.G. Johnson, M.A. Robb, J.R. Cheeseman, T. Keith, G.A. Peterson, J.A. Montgomery, K. Raghavachari, M.A. AI-Laham, V.G. Zakrzewski, J.V. Ortiz, J.B. Foresman, J. Ciolowski, B.B. Stefanov, A. Nanayakkara, M. Challacombe, C. Y. Peng, P. Y. Ayala, W. Chen, M.W. Wong, J.L. Andres, E.S. Replogle, R. Gomperts, R. L. Martin, D.J. Fox, J.S. Binkley, D.J. Defrees, J. Baker, J.P. Stewart, M. Head-Gordon, C. Gonzalez, J.A. Pople, Gaussian, Inc., Pittsburgh, PA, 1995.

[15] http://laaksonen.csc.fi/gopenmolI.

[16] G.E. Walrafen, D.M. Dodd, Trans. Faraday Soc. 57 (1961) 1286.

[17] P.A. Giguere, R. Svoie, J. Am. Chem. Soc. 85 (1963) 287.

[18] S.J. Cyvin, Acta Chem. Scand. 24 (1970) 1499.

[19] D.R. Sull, H. Prophet (Eds.), Natl. Ref Data Ser., Natl. Bur. Sumd., No. 37 (1971).

[20] K.B. Snow, T.F. Thomas, Int. J. Mass Spectrom. Ion. Proc. 96 (1990) 49.

[21] R.K. Chang, J.H. Eickmans, W-F. Hsieh, C.F. Wood, J-Z. Zhang, J-B. Zheng, Appl. Optics 27 (1988) 2377.

[22] B. Mason, The Earth as a Planet, G.P. Kuiper (Ed.), University of Chicago Press, Chicago, 1954, p. 267.

[23] V.A. Krasnopolsky, Photochemistry of the Atmospheres of Mars and Venus, Springer, Berlin, 1986, pp. 159-172. 